\title{
An Extreme Point Result for Robust Stability of Discrete-Time Interval Polynomials*
}

\author{
B. Ross Barmish
}

Department of Electrical and Computer Engineering

University of Wisconsin-Madison

Madison, Wisconsin 53706

\section{Abstract}

For the case of discrete-time interval polynomials, the attainment of Kharitonov-like extreme point results has been focal point of recent attention. To date, the strongest available result is applicable subject to the restriction that the coefficients of $z^{k}$ are fixed for $k>\left\lfloor\frac{n}{2}\right\rfloor$ where $n$ is the degree of the interval polynomial. This paper gives conditions under which perturbations can be tolerated in the higher order coefficients while preserving the property that stability of the extreme polynomials implies stability of the entire family.

\section{Introduction and Formulation}

Motivated by the seminal theorem of Kharitonov (1978) for the continuous-time case, a number of authors have investigated the extent to which one can obtain analogous results in discrete-time; see Jury (1988) for a survey. This is also the topic of this paper. by

We consider to the (discrete-time) interval polynomial denoted

$$
p(z, q)=\sum_{i=0}^{n}\left[q_{i}^{-}, q_{i}^{+}\right] z^{i}
$$

By this notation, we mean a polynomial of the form

$$
p(z, q)=\sum_{i=0}^{n} q_{i} z^{i}
$$

where

$$
q_{i}^{-} \leq q_{i} \leq q_{i}^{+}
$$

for $i=0,1, \cdots, n$ and $q_{i}^{-}$and $q_{i}^{+}$are given bounds. We say that $p(z, q)$ is robustly stable if for all admissible $q \doteq\left(q_{0}, q_{1}, q_{2}, \cdots, q_{n}\right)$ satisfying (1), the roots of $p(z, q)$ lie in the open unit disk.

The Issue of Kharitonov-like Results: A number authors have considered the following problem: Let $q^{i}$ denote the $i$-th extreme point of the box m.

$$
Q \doteq\left\{\left(q_{0}, q_{1}, \ldots, q_{n}\right) \in \mathbf{R}^{n+1}: q_{i}^{-} \leq q_{i} \leq q_{i}^{+} \text {for } i=0,1,2, \cdots, n\right\} .
$$

Give conditions under which stability (all roots in the open unit disk) of all the extreme polynomials $p\left(z, q^{i}\right)$ implies robust stability of $p(z, q)$. This being the case, a weak Kharitonov Theorem is said to hold.

It is already known that some sort of conditions must be imposed on $p(z, q)$ in order to obtain a weak Kharitonov theorem. Otherwise, counterexamples along the lines of Bose and Zeheb (1987), Hollot and Bartlett (1986) and Cieslik (1987) come into play. In Kraus, Anderson and Mansour (1987), $Q$ is replaced by a type of "rotated" rectangle as a means for obtaining an extreme point result. This result is further strengthened in Mansour, Kraus and Anderson (1988).

The paper of Hollot and Bartlett (1986), however, gives the strongest result to date within the more traditional box framework for $Q$. It is shown that if $q_{i}^{-}=q_{i}^{+}$for $i>\left\lfloor\frac{n}{2}\right\rfloor$, then a weak Kharitonov theorem holds. That is, if one only allows uncertainty in approximately half of the polynomial coefficients (the lower order coefficients), then stability of the extreme polynomials $p\left(z, q^{i}\right)$ implies robust stability of $p(z, q)$.

*This work was supported by the National Science Foundation under Grant No. ECS-8612948.
In this paper, conditions are given under which one can obtain a bonus beyond $\left\lfloor\frac{n}{2}\right\rfloor$. To this end, we define a certain maximal perturbation index $i_{\max }$ which has some important properties: First, for any interval polynomial,

$$
i_{\max } \geq\left\lfloor\frac{n}{2}\right\rfloor
$$

Second, a weak Kharitonov theorem holds provided

$$
q_{i}^{-}=q_{i}^{+}
$$

for $i>i_{\text {max }}$. That is, we require a smaller number of fixed coefficients than in the existing literature.

The technical novelty of this paper is that we take the root distribution of the extreme polynomials $p\left(z, q^{i}\right)$ into account. It will be seen that "good" root distributions lead to higher values of $i_{\max }$ than "bad distributions." Roughly speaking, the most desirable situation occurs when all extreme polynomials have their roots on the same side of the imaginary axis and are close to $z=0$. Letting $r$ denote a confinement radius for the roots, it turns out that $i_{\text {max }}$ increases as $r$ decreases.

\section{Definition: The Maximal Perturbation Index $i_{\max }$}

Consider the discrete-time interval polynomial $p(z, q)$ with assocjated set of extreme polynomials $\left\{p\left(z, q^{i}\right)\right\}$ and suppose that $r \in[0,1)$ is given. For $p\left(z, q^{i}\right)$, let $n_{L, i}$ be the number of roots in the semi-open left half of the disk of radius $r$

$$
\mathcal{D}_{L, r} \doteq\{z \in \mathbf{C}: \text { Re } z \leq 0 \text { and }|z| \leq r\}
$$

and define the left index of $p\left(z, q^{i}\right)$ to be the smallest of the $n_{L, i}$; i.e.,

$$
n_{L} \doteq \min _{i} n_{L, i}
$$

Similarly, for $p\left(z, q^{i}\right)$, let $n_{R, i}$ be the number of roots in the semiopen right half of the disk of radius $r$

$$
\mathcal{D}_{R, r} \doteq\{z \in \mathrm{C}: \operatorname{Re} z \geq 0 \text { and }|z| \leq r\}
$$

and define the right index of $p\left(z, q^{i}\right)$ to be the smallest of the $n_{R, i}$; i.e.,

$$
n_{R} \doteq \min _{i} n_{R, i}
$$

Now, with notation as above, the maximal perturbation index is defined by

$i_{\max } \doteq \begin{cases}\left\lfloor\frac{n}{2}\right\rfloor+\max \left\{\left\lfloor\frac{n_{L}(1-r)}{2(1+r)}\right\rfloor,\left\lfloor\frac{n_{R}(1-r)}{2(1+r)}\right\rfloor\right\} & \text { for } n \text { even } \\ \left\lfloor\frac{n}{2}\right\rfloor+\max \left\{\left\lfloor\frac{n_{L}(1-r)}{2(1+r)}+\frac{1}{2}\right\rfloor,\left\lfloor\frac{n_{R}(1-r)}{2(1+r)}+\frac{1}{2}\right\rfloor\right\} & \text { for } n \text { odd. }\end{cases}$

For notational simplicity, we do not explicitly show the dependence of $n_{L}, n_{R}$ and $i_{\max }$ on $r$.

\section{Main Result}

In the theorem to follow, we impose two nontriviality conditions. Namely, it is assumed that 


$$
\operatorname{sign}\left(\sum_{i=0}^{n} q_{i}^{-}\right)=\operatorname{sign}\left(\sum_{i=0}^{n} q_{i}^{+}\right)
$$

and

$$
\operatorname{sign}\left(\sum_{i \text { even }} q_{i}^{-}+\sum_{i \text { odd }} q_{i}^{+}\right)=\operatorname{sign}\left(\sum_{i \text { even }} q_{i}^{+}+\sum_{i \text { odd }} q_{i}^{-}\right) .
$$

Discussion of Nontriviality Conditions: Note that there is no loss of generality in imposing condition (3). That is, if (3) violated, it follows that

$$
\sum_{i=0}^{n} q_{i}^{-} \leq 0 \leq \sum_{i=0}^{n} q_{i}^{+} .
$$

This inequality implies that $p\left(1, q^{*}\right)=0$ for some $q^{*} \in Q$. Similarly, if (4) is violated, it follows that $p\left(-1, q^{*}\right)=0$ for some $q^{*} \in Q$. In either case, we contradict robust stability. We are now prepared to state the main result.

Theorem 1: Consider the discrete-time interval polynomial $p(z, q)$ satisfying the nontriviality conditions (3) and (4) and assume further that for fixed $r \in[0,1), q_{i}^{-}=q_{i}^{+}$for $i>i_{\max }$. Then, $p(z, q)$ is robustly stable if and only if the extreme polynomials $p\left(z, q^{i}\right)$ are stable.

\section{Examples Illustrating Application of Theorem 1}

Consider the discrete-time interval polynomial

$$
p(z, q)=10 z^{3}+[-3,-2.8] z^{2}+0.4 z-0.02
$$

and notice that $\left\lfloor\frac{n}{2}\right\rfloor=1$ and $q_{2}^{-} \neq q_{2}^{+}$. Hence, the $\left\lfloor\frac{n}{2}\right\rfloor$ result does not apply. We now compute $i_{\max }$. Indeed, the roots of the first extreme polynomial $p(z,-3)$ are $z_{1,2} \approx 0.1 \pm j 0.1$ and $z_{3}=0.1$ and the roots of the second extreme polynomial $p(z,-2.8)$ are $z_{1,2} \approx 0.097 \pm j 0.118$ and $z_{3}=0.086$. It is easy to verify that with $r \approx 0.15$, we obtain $n_{L}=0, n_{R}=3$ and

$$
i_{\max }=\left\lfloor\frac{3}{2}\right\rfloor+\left\lfloor\frac{3(1-0.15)}{2(1+0.15)}+\frac{1}{2}\right\rfloor=2
$$

Noting that $q_{3}^{-}=q_{3}^{+}=10$, Theorem 1 applies and we conclude that $p(z, q)$ is robustly stable.

A second interesting example is the discrete-time interval polynomial

$$
p(z, q)=z^{4}+\left[-\frac{17}{8}, \frac{17}{8}\right] z^{3}+\frac{3}{2} z^{2}-\frac{1}{3}
$$

considered by Bose and Zeheb (1987). These authors demonstrate that the two extreme polynomials $p\left(z, q^{1}\right)$ and $p\left(z, q^{2}\right)$ are strictly stable but $p(z, q)$ is not robustly stable. We now interpret this result in terms of Theorem 1 above. Indeed, it is easily verified that $p\left(z, q^{1}\right)$ and $p\left(z, q^{2}\right)$ each have 3 roots which are "close" to the unit circle (within a distance of 0.1 ). The fourth root of $p\left(z, q^{1}\right)$ is located at $z \approx-0.37$ and the fourth root of $p\left(z, q^{2}\right)$ is located at $z \approx 0.37$. Hence, the obvious choice is $r \approx 0.37$. This leads to $n_{L}=n_{R}=0$ which implies that $i_{\max }=2$. This "low" value of $i_{\max }$ is synergistic with Bose and Zeheb's conclusion.

\section{Discussion}

The objective of this section is to provide some intuitive feeling as to what kind of root distributions for the $p\left(z, q^{i}\right)$ are "good" as far as maximality of $i_{\max }$ is concerned. For simplicity, we only consider $n$ even and note that the discussion is trivially modified to handle $n$ odd.

The first case we consider is when $r \rightarrow 0$ and all roots of the $p\left(z, q^{i}\right)$ have the same sign on their real parts. Then $i_{\max } \rightarrow n$. That is, if all the roots of the extreme polynomials are "clustered" on the same side of the imaginary axis near $z=0$, then in the limit, a weak Kharitonov Theorem holds without any fixed coefficient constraints.
To further explore the relationship between $i_{\max }$ and the root confinement radius $r$, suppose $p(z, q)$ is monic and again, all roots of the $p\left(z, q^{i}\right)$ are on the same side of the imaginary axis. For example, this situation can easily occur when all the differences $q_{i}^{+}-q_{i}^{-}$are small-small perturbations about a stable polynomial. Now, we want to know how small $r$ must be so that perturbations in all coefficients can be tolerated while a weak Kharitonov Theorem holds. Then, setting $i_{\max } \geq n-1$, we use (2) to obtain the condition

$$
\frac{n}{2}+\left\lfloor\frac{n(1-r)}{2(1+r)}\right\rfloor>n-1
$$

which is guaranteed if

$$
r \leq \frac{1}{n-1}
$$

Using the $i_{\text {max }}$ formula, it is possible to explore many other tradeoffs. For example, if one only wishes to handle perturbations up to and including the coefficient of $z^{n-1}$, then a similar analysis leads to

$$
r \leq \frac{2}{n-2}
$$

\section{Interpretations and Improvements on the $i_{\max }$ Formula}

In the full version of the paper (see Barmish (1989)), additional interpretations of the $i_{\max }$ formula are provided. It is also seen that the $i_{\text {max }}$ formula can be improved by taking more detailed information about the root distribution of the $p\left(z, q^{i}\right)$ into account. The simplest improvement on the $i_{\max }$ formula is motivated by the fact that $i_{\text {max }}$ in (2) takes the magnitude of the roots into account but not the phase. By creating "confinement sectors" for the roots, a new $i_{\max }$ formula is derived which depends not only on the confinement radius $r$ but also on the confinement angle $\phi ; i_{\text {max }}$ increases as $\phi$ decreases.

\section{References}

Barmish, B. R. (1989). Full version of this paper, Technical Report ECE-89-10, Department of Electrical and Computer Engineering, University of Wisconsin-Madison.

Bose, N. K. and E. Zeheb (1986). Kharitonov's Theorem and Stability Test of Multidimensional Digital Filters, IEE Proceedings Pt. G, vol. 133 , no. 4 , pp. 187-190.

Cieslik, J. (1987). On the Possibilities of the Extension of Kharitonov's Stability Test for Interval Polynomials to the Discrete-Time Case, IEEE Transactions on Automatic Control, AC-32, pp. 237-238.

Hollot, C. V. and A. C. Bartlett (1986). Some Discrete-Time Counterparts to Kharitonov's Stability Criterion for Uncertain Systems, IEEE Transactions on Automatic Control, AC-31, pp. 355-356.

Jury, E. I. (1988). Robustness of Discrete Systems: A Review, to appear.

Kharitonov, V. L. (1978). Asymptotic Stability of an Equilibrium Position of a Family of Systems of Linear Differential Equations, Differential'nye Uraveniya, vol. 14, no. 11, pp. 1483-1485.

Kraus, F., B. D. O. Anderson and M. Mansour (1987). Robust Schur Polynomial Stability and Kharitonov's Theorem, Proceedings of IEEE Conference on Decision and Control, Los Angeles.

Mansour, M., F. Kraus and B. D. O. Anderson (1988). Strong Kharitonov Theorem for Discrete Systems, Proceedings of IEEE Conference on Decision and Control, Austin. 